\title{
Cross-layer Design for Optimizing TCP Performance
}

\author{
A. Chockalingam ${ }^{\dagger}$, Eitan Altman $^{\ddagger}$, J. V. K. Murthy ${ }^{\dagger}$, and Ramdoot Kumar ${ }^{\dagger}$ \\ $\dagger$ Department of ECE, Indian Institute of Science, Bangalore 560012, India \\ $\ddagger$ INRIA B.P.93, 2004 Route des Lucioles 06902 Sophia-Antipolis Cedex, France
}

\begin{abstract}
In this paper, we propose and analyze novel and efficient cross-layer designs involving joint optimization of physical, link, and TCP layers in wireless. Particularly, we investigate the design of symbol mapping diversity (SMD) schemes using $M$ QAM at the physical layer for optimal goodput performance at the TCP layer. We present the design and TCP goodput analysis of two SMD schemes, one applying SMD at the TCP packet level, termed as Full Packet SMD (FP SMD), and the other applying SMD at the link layer (LL) packet level, termed as $L L$ ARQ SMD. We show that although the LL ARQ SMD scheme offers good TCP goodput at high SNRs, it performs poorer compared to FP SMD scheme at low SNRs because of increased delays incurred due to increased $L L$ retransmissions at low SNRs. We therefore propose and analyze a hybrid SMD scheme which adaptively switches modes (between LL ARQ SMD and FP SMD) based on measured LL packet error rate. We show that the hybrid SMD scheme combines the best TCP performance of both LL ARQ SMD and FP SMD under varying channel conditions.
\end{abstract}

Keywords - M-QAM, symbol mapping diversity, ARQ, TCP, goodput.

\section{INTRODUCTION}

Higher order modulation (e.g., $M$-QAM, $M$-PSK) and packet retransmissions (e.g., ARQ) are popular techniques to achieve high data rates and low error rates, respectively, on wireless channels. Recently, a packet combining method known as symbol mapping diversity (SMD) has been investigated for $M$-ary modulation on wireless channels [1],[2]. Symbol mapping diversity involves varying the bit-to-symbol mapping in $M$-ary modulation across multiple (re)transmissions of the same packet. This results in improved packet combining performance in terms of reduced packet error rates compared to multiple (re)transmissions schemes without SMD. This improved physical layer performance due to SMD is desired for achieving good performance at higher layers like TCP. However, multiple (re)transmissions of the same packet in SMD introduce additional delays (i.e., increased round-trip time, RTT), which can hurt TCP performance. Hence, cross-layer designs involving joint optimization of the physical, link, and TCP layers are desired. Our focus in this paper is the joint design of SMD at the physical layer and ARQ at the link layer for optimal goodput performance at the TCP layer.

Specifically, we consider the design and TCP goodput analysis of two SMD schemes using $M$-QAM; one applying SMD at the TCP packet level, termed as Full Packet SMD (FP SMD), and the other applying SMD at the link layer (LL) packet level, termed as LL ARQ SMD. We show that although the LL ARQ SMD scheme offers good TCP goodput at high SNRs, it performs poorer compared to FP SMD scheme at low SNRs because of increased delays incurred due to increased LL retransmissions at low SNRs. We therefore

This work was supported in part by the Indo-French Centre for Promotion of Advanced Research, New Delhi, under Project 2900-IT.

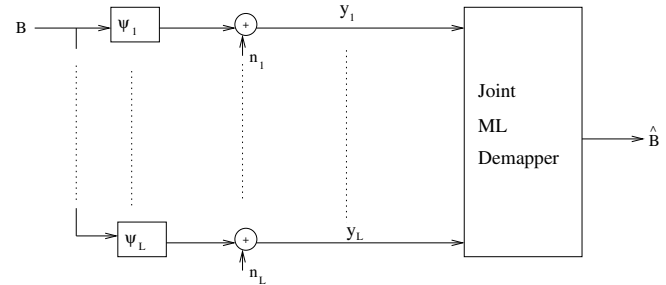

Fig. 1. Symbol mapping diversity (SMD) scheme

propose and analyze a hybrid SMD scheme which adaptively switches modes (between LL ARQ SMD and FP SMD) based on measured LL packet error rate. We show that the hybrid SMD scheme combines the best TCP performance of both LL ARQ SMD and FP SMD under varying channel conditions.

\section{SyMbOL MAPPING DIVERSITY}

In an $M$-ary modulation scheme, a bit block $B$ consisting of $m_{q}=\log _{2} M$ bits are taken and mapped to a point in the signal constellation via a bit-to-symbol mapping function $\psi$, and this signal point $\psi(B)$ is transmitted on the channel. The number of possible bit-to-symbol mappings are $M$ !. In order to achieve packet combining diversity, the same bits may be transmitted more than once. Let $L$ be the number of such transmissions. Multiple transmissions of the same data block $B$ can either use the same bit-to-symbol mapping in all transmissions, or vary the bit-to-symbol mapping in each transmission; we call the former scheme as the maximum-likelihood combining diversity (MLD) scheme and the latter scheme as the symbol mapping diversity (SMD) scheme.

Figure 1 shows the SMD scheme where the same bit block $B$ is sent $L$ times using $L$ different mappings $\psi_{1}, \psi_{2}, \cdots, \psi_{L}$. Assuming AWGN, the receiver obtains the received samples

$$
y_{i}=\psi_{i}(B)+n_{i}, \quad i=1,2, \cdots, L,
$$

where $n_{i}=n_{i I}+j n_{i Q}$ is a complex Gaussian r.v of zero mean and variance $\sigma^{2} / 2$ per dimension. The received samples $y_{i}$ 's are combined at the receiver to make an estimate of the transmitted bits, $\widehat{B}$. Given the observations $y_{1}, y_{2}, \cdots, y_{L}$ in (1), the receiver decides that the data block $B$ was transmitted according to the ML decision rule

$$
\widehat{B}=0,1, \cdots, M-1 \sum_{i=1}^{\min }\left|y_{i}-\psi_{i}(\widehat{B})\right|^{2} .
$$

For the SMD scheme, a key question is how to obtain the optimum mappings $\psi_{1}, \psi_{2}, \cdots, \psi_{L}$. One way is to obtain expressions for the bit error rate (BER) or symbol error rate (SER) of the SMD scheme and choose the mappings that minimize the BER/SER. We consider $M$-QAM for which we obtain the optimum mappings by minimizing the following upper bound on the SER of the SMD scheme with $L$ transmissions [1]: 


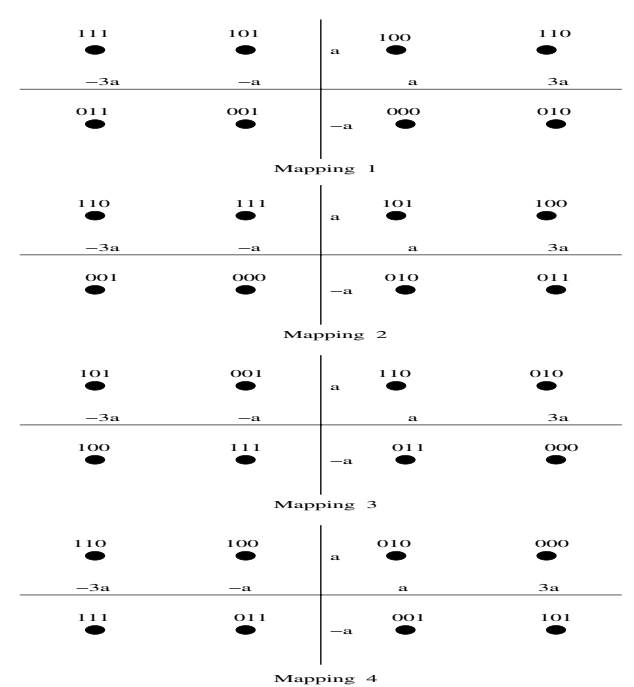

Fig. 2. Bit-to-symbol mappings obtained by minimizing the SER upper bound. $\psi_{1}$ : Mapping 1, $\psi_{2}$ : Mapping 2, $\psi_{3}$ : Mapping 3, $\psi_{4}$ : Mapping 4.

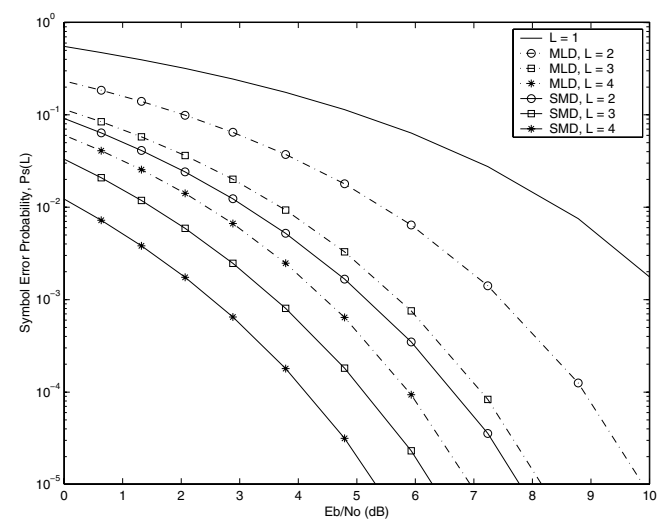

Fig. 3. Symbol error probability, $P_{s}(L)$, versus $E_{b} / N_{o}$ for SMD and MLD schemes. $L=1,2,3,4$.

$$
P_{s}(L) \leq \frac{1}{M} \sum_{B=0}^{M-1} \sum_{\substack{C=0 \\ C \neq B}}^{M-1} Q\left(\sqrt{\frac{1}{4 \sigma^{2}} \sum_{i=1}^{L} \mathcal{D}^{2}\left[\psi_{i}(B), \psi_{i}(C)\right]}\right)
$$

where $\mathcal{D}[x, y]$ is the Euclidean distance between points $x$ and $y$. We use 8-QAM for the purpose of illustrating different design examples of optimizing TCP goodput. Accordingly, we obtained the optimum mappings for 8-QAM that minimize the SER bound in (3). The resulting optimum mappings $\psi_{1}, \psi_{2}, \psi_{3}, \psi_{4}$ are shown in Fig. 2.

In Fig. 3, we plot the SER bound as per (3) as a function of $E_{b} / N_{o}$ for the MLD and SMD schemes for $L=2,3,4$. Performance without MLD/SMD (i.e., $L=1$ ) is also shown. The first mapping $\psi_{1}$ is used in all the $L$ transmissions in the MLD scheme. From Fig. 3, it is seen that the SMD scheme provides better SER performance than the MLD scheme because of the improved packet combining benefit in SMD.

\section{SMD FOR OPTIMAL TCP PERFORMANCE}

In this section, we present the design and analysis of two SMD schemes for optimal TCP performance. In one scheme, SMD is applied at the TCP packet level, and in the other scheme SMD is applied at the LL packet level; we call the former as the full packet SMD (FP SMD) scheme and the latter as LL ARQ scheme.

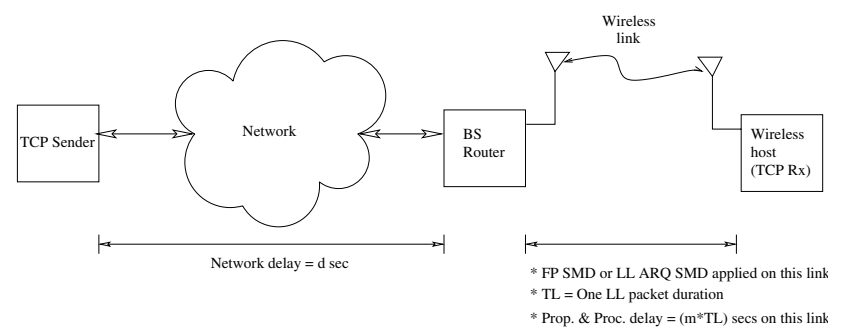

Fig. 4. Wireless network model

The wireless network model considered is shown in Fig. 4. The TCP sender is on a host on the network side and the TCP receiver is on a wireless host. The wireless host is connected to the network through a router at the base station (BS). The network delay between the TCP sender and the BS router is $d$ secs. We will assume error-free feedback from the receiver to the sender. FP SMD or LL ARQ SMD is applied on the packet (re)transmissions on the wireless link.

\section{A. Full Packet SMD Scheme}

In this scheme, the entire TCP packet is repeated $L$ times using SMD on the wireless link (with a motivation to improve the TCP packet error rate on the wireless link by exploiting the SMD benefit at the physical layer as illustrated in Sec. II). There is no link layer protocol in this scheme. TCP packet size is $N$ bits. Each TCP packet transmission corresponds to $N L$ transmitted bits. We consider coding using some code $c \in \mathcal{C}$, where $\mathcal{C}$ is some set of codes. More precisely, assume that the $N$-bit TCP packet is constructed by $N / m_{q}$ QAM symbols where each QAM symbol contains $m_{q}=\log _{2} M$ bits; the packet contains $K=K(c)$ useful information bits (or $K / m_{q}$ useful QAM symbols) plus $R(c)=(N-K(c)) / m_{q}$ redundant QAM symbols. We assume that a $(n, k)$ Reed-Solomon (RS) block code is used so that up to $t$ errored RS code symbols in a block of $n$ RS code symbols can be corrected, where $t$ is given by $t=(n-k) / 2$ for $(n-k)$ even and $t=(n-k-1) / 2$ for $(n-k)$ odd.

Our interest is to choose $L$ that maximizes the TCP goodput. For this we need an expression for TCP throughput/goodput. We will use the well known 'square-root formula' for TCP throughput [3]. The delay model and TCP packet loss model are presented below.

Delay model: If transmission of bits is continuous at a constant rate $\mu$ packets/sec and the network delay is $d$ secs, then

$$
R T T(L)=2 d+L / \mu
$$

Note: We neglected the transmission time of a TCP ACK which is to be much shorter than the transmission time of a TCP data packet.

TCP packet loss model: Assuming independent QAM symbol errors, the RS code symbol error probability for code $c$, $P_{r s c}(L, c)$, is

$$
P_{r s c}(L, c)=1-\left(1-P_{s}(L)\right)^{l_{r}} \text {, }
$$

where $l_{r}$ is the number of QAM symbols per RS code symbol. The RS code block error probability for code $c, P_{b l o c k}(L, c)$, is then given by

$$
P_{\text {block }}(L, c)=\sum_{i=t+1}^{n}\left(\begin{array}{c}
n \\
i
\end{array}\right)\left[P_{r s c}\left(L_{R}, c\right)\right]^{i}\left[1-P_{r s c}(L, c)\right]^{n-i} \text {. (6) }
$$


The TCP packet loss probability, $P_{\text {loss }}(L, c)$, is then

$$
P_{\text {loss }}(L, c)=1-\left(1-P_{\text {block }}(L, c)\right)^{X},
$$

where $X$ is the number of RS code blocks per TCP packet.

Optimizing the TCP goodput: Our interest is to choose $L$ that maximizes the TCP goodput. TCP throughput in packets per second is given by [3]

$$
\eta(L, c)=\min \left(\frac{1}{R T T(L, c)} \sqrt{\frac{\alpha}{P_{\text {loss }}(L, c)}}, \frac{\mu}{L}\right)\left(1-P_{\text {loss }}(L, c)\right),
$$

and the TCP goodput is given by

$$
\operatorname{Gput}(L, c)=\left(\frac{K(c)-H_{T}}{N}\right) \eta(L, c)
$$

where $H_{T}$ is the number of bits in the TCP header. The constant $\alpha$ equals 1.5 if the delayed ACK mechanism is disabled and equals 0.75 if it is enabled. Note that if $p_{s}(L)$ stands for an upper bound for the QAM symbol error probability (as in (3)), we maximize in fact a lower bound on the TCP goodput. For the delay model in (4), we obtain the problem of maximizing

$$
\begin{aligned}
\operatorname{Gput}(L, c)= & \left(\frac{K(c)-H_{T}}{N}\right) \times \min \left(\frac{1}{2 d+L / \mu} \sqrt{\frac{\alpha}{P_{\text {loss }}(L, c)}}, \frac{\mu}{L}\right) \\
& \times\left(1-P_{\text {loss }}(L, c)\right) .
\end{aligned}
$$

1) Design Example and Performance: In this subsection, we present a design example of the FP SMD scheme and its performance. We consider the following system parameters: \# bits per TCP packet, $N=12000$ bits; $\alpha=1.5$ \# header bits in a TCP packet, $H_{T}=160$ bits \# bits per QAM symbol, $m_{q}=3$ (i.e., 8-QAM) \# bits per RS code symbol, $m_{r}=6$ (i.e., $l_{r}=2$ ) $(42,34)$ RS block code network delay $d$, measured in \# of TCP packets, $q=10$.

We observed that the TCP packet loss probability, $P_{\text {loss }}(L, c)$, decreases as the number of repetitions of a TCP packet, $L$, is increased (graph not shown due to space limitation). However, increasing $L$ also implies an increase in the $R T T$ which is equal to $2 d+L / \mu$ (as per (4)). In Fig. 5, we plot the TCP goodput as a function of $E_{b} / N_{o}$ for the FP SMD scheme with $L=1,2,3,4$.

The following observations can be made from Fig. 5. It can be seen that the optimum value of $L$ depends on the operating SNR of the link. For example, for $E_{b} / N_{o}>9 \mathrm{~dB}$ (i.e., high SNRs), $L=1$ (i.e., no multiple transmissions) is the optimum choice as it achieves the best normalized TCP goodput of $\left(K(c)-H_{T}\right) / N$. This is because $P_{\text {loss }}$ becomes small enough at these high SNRs and increasing $L$ will increase the RTT (as per (4)) which reduces the TCP goodput. For $L>1$, the maximum achievable TCP goodput gets reduced by a factor of $L$. For SNRs in the range $4 \mathrm{~dB}$ to $8 \mathrm{~dB}, L=2$ (not $L=1$ ) happens to be the optimum choice; this is because the $P_{\text {loss }}$ gain achieved by $L=2$ compared to $L=1$ dominates in the throughput expression than the $R T T$ increase incurred by $L=2$ compared to $L=1$. The maximum achieved normalized goodput in this case is $\left(K(c)-H_{T}\right) / 2 N$. Likewise, for the SNRs in the range 2 $\mathrm{dB}$ to $3 \mathrm{~dB}$, the optimum choice of $L$ is 3 with a maximum

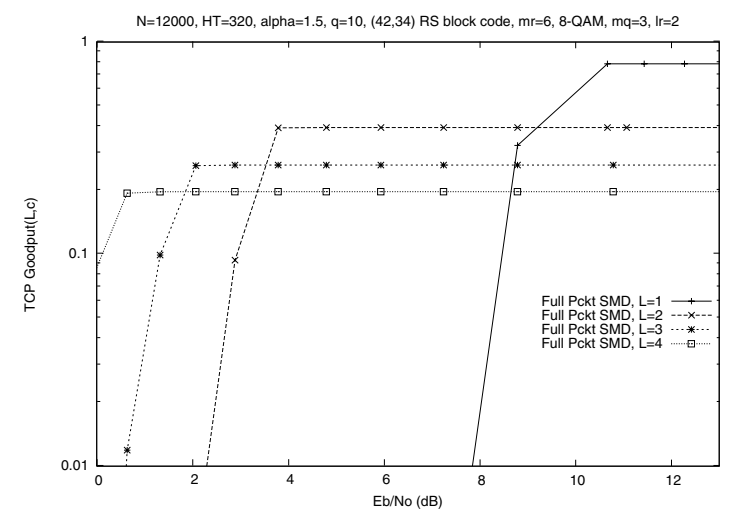

Fig. 5. TCP Goodput versus $E_{b} / N_{0}$ for FP SMD scheme. $N=12000$ bits, $H_{T}=160$ bits, (42,34) RS code, $m_{r}=6$ bits, 8-QAM, $m_{q}=3$ bits, $l_{r}=2 . L=1,2,3,4$.

achievable goodput of $\left(K(c)-H_{T}\right) / 3 N$. Thus, Fig. 5 enables the designer to choose the optimum value of $L$ that maximizes the TCP goodput for the operating SNR of the link.

Although FP SMD with $L>1$ improves the TCP goodput at low SNRs compared to $L=1$, it looses out on the maximum achievable goodput at high SNRs (limited by $\mu / L$ ) which is not desirable. This motivates the use of an ARQ at the link layer (LL) and apply SMD only on erroneous LL packets instead of on all full TCP packets. Accordingly, we propose and analyze an LL ARQ SMD scheme in the following.

\section{B. LL ARQ SMD Scheme}

Here, we consider a system which uses an ARQ at the link layer (LL) on the wireless segment in order to improve the error rate seen by the TCP layer. Again, consider the system model shown in Fig. 4. The BS router takes each TCP packet (assumed to be of fixed size of $N$ bits, which includes $H_{T}$ bits of TCP header) and segments it into $X_{L}$ equal sized LL packets which are sent over the wireless link. Each LL packet is assumed to consist of $X_{B}$ number of $(n, k)$ RS coded blocks. A LL ARQ runs between the BS and the wireless host. The LL ARQ attempts to recover erroneous LL packets by allowing up to a maximum of $L_{R}-1$ retransmissions (i.e., $L_{R}$ transmissions including the first transmission of the LL packet). The number of overhead bits (for the purpose of LL $\mathrm{CRC}, \mathrm{ARQ}$ sequence numbering, etc) per LL packet is taken to be $H_{L}$ bits. The propagation \& processing delay on the wireless link is assumed to occupy an integer number of LL packets duration, i.e., ACK/NACK for a LL packet is available at the sender $m$ LL packets duration after that LL packet is sent. We also assume error-free LL ARQ feedback. SMD is used on the LL packet retransmissions; i.e., different bitto-symbol mappings are used for (up to) $L_{R}$ different transmissions of the same LL packet. We consider a stop-and-wait ARQ here. However, Go-back-N and Selective Repeat type ARQs can also be considered likewise.

With the above model and assumptions, expressions for $R T T$ and $P_{\text {loss }}$ for the LL ARQ SMD can be obtained as follows. The RS code symbol error probability, $P_{r s c}\left(L_{R}, c\right)$, is given by

$$
P_{r s c}\left(L_{R}, c\right)=1-\left(1-P_{s}\left(L_{R}\right)\right)^{l_{r}}
$$

where $P_{s}\left(L_{R}\right)$ is the QAM symbol error probability with 
SMD given by (3). The RS code block error probability, $P_{\text {block }}\left(L_{R}, c\right)$, is then given by

$$
P_{\text {block }}\left(L_{R}, c\right)=\sum_{i=t+1}^{n}\left(\begin{array}{c}
n \\
i
\end{array}\right)\left[P_{r s c}\left(L_{R}, c\right)\right]^{i}\left[1-P_{r s c}\left(L_{R}, c\right)\right]^{n-i} \text {. }
$$

Expression for RTT: Because of retransmissions in the LL ARQ, the effective length of a TCP packet on the wireless link increases depending on the LL packet error probability, $L_{R}$ and $m$. We are interested in finding the average effective length of a TCP packet.

Let $Y$ denote the number of times a given LL packet is likely to be sent ( $1 \leq Y \leq L_{R}$ ). For the case of $L_{R}=1$ (i.e., no ARQ in case of error), $\operatorname{pr}(Y=i)$ is equal to one for $i=L_{R}$ and zero otherwise. For the case of $L_{R}>1$,

$$
\operatorname{pr}(Y=i)= \begin{cases}1-P_{L L}(i, c), & i=1 \\ \left(1-P_{L L}(i, c)\right) \prod_{j=1}^{i-1} P_{L L}(j, c), & 1<i<L_{R} \\ \prod_{j=1}^{i-1} P_{L L}(j, c), & i=L_{R} \\ 0, & i>L_{R} .\end{cases}
$$

where $P_{L L}(j, c)$ is the probability that the $j$ th attempt of a LL packet is in error, which, in terms of the RS code block error probability, can be written as

$$
P_{L L}(j, c)=1-\left(1-P_{\text {block }}(j, c)\right)^{X_{B}},
$$

where $P_{b l o c k}(j, c)$ is given by (12). Let $L_{i}$ denote the effective length of the attempt $i$ (measured in number of LL slots), which can be obtained as

$$
L_{i}=1+(i-1)(m+1), \quad 1 \leq i \leq L_{R} .
$$

With the above, the average effective length of one TCP packet, $L_{e f f}$, normalized by the number of LL packets $X_{L}$, is

$$
L_{e f f}\left(L_{R}, c\right)=\sum_{i=1}^{L_{R}} L_{i} \cdot \operatorname{pr}(Y=i),
$$

The average $R T T$ is then given by

$$
R T T\left(L_{R}, c\right)=2 d+\frac{L_{e f f}\left(L_{R}, c\right)}{\mu} .
$$

It can be noted that the maximum achievable TCP throughput in this LL ARQ SMD scheme is $\mu / L_{e f f}\left(L_{R}, c\right)$, instead of $\mu / L$ as in the case of FP SMD scheme.

Expression for $P_{\text {loss }}$ : The expression for TCP packet error probability, $P_{\text {loss }}$, for this LL ARQ SMD scheme can be obtained as follows. Noting that $X_{L}$ is the number of LL packets per TCP packet and defining

$$
p \triangleq \prod_{j=1}^{L_{R}} P_{L L}(j, c)
$$

$P_{\text {loss }}^{\left(X_{L}\right)}\left(L_{R}, c\right)$ can be obtained using the recursive relation

$$
P_{\text {loss }}^{\left(X_{L}\right)}\left(L_{R}, c\right)=p(1-p)^{X_{L}-1}+P_{\text {loss }}^{\left(X_{L}-1\right)}\left(L_{R}, c\right),
$$

with the boundary condition $P_{\text {loss }}^{(0)}\left(L_{R}, c\right)=0$.

The $P_{\text {loss }}^{\left(X_{L}\right)}\left(L_{R}, c\right)$ expression in (19) along with the $R T T$ expression in (17) can be substituted in the TCP square root formula to obtain the for TCP throughput/goodput.

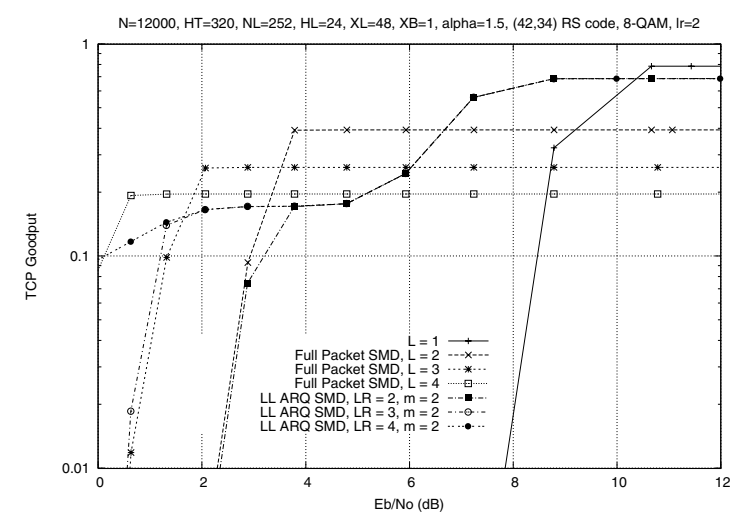

Fig. 6. TCP goodput versus $E_{b} / N_{o}$ for $a$ ) FP SMD, and $b$ ) LL ARQ SMD. $N=12000$ bits. $H_{T}=160$ bits. $N_{L}=252$ bits. $H_{L}=24$ bits. $X_{L}=48$ LL packets. $X_{B}=1$ block. $\alpha=1.5 . q=10$. $(42,34)$ RS block code. 8-QAM. Prop. \& proc. delay slots on the wireless link, $m=2$.

1) Design Example and Performance: In this subsection, we present a design example of the LL ARQ SMD and its performance. In addition to the system parameters assumed in Sec. III-A.1, we consider the following LL parameters:

\# bits per LL packet, $N_{L}=252$ bits

\# overhead bits in a LL packet, $H_{L}=24$ bits

\# LL packets per TCP packet, $X_{L}=\left\lceil\frac{N}{N_{L}}\right\rceil=48$

\# RS coded blocks per LL packet, $X_{B}=\frac{N_{L}}{n m_{r}}=1$.

We observed that for the same $L, L_{R}>1$, the LL ARQ SMD scheme achieves better $P_{\text {loss }}$ performance than the FP SMD scheme (graph not shown due to space limitation). However, the RTT in LL ARQ SMD can be larger than that in FP SMD for large $m$, depending on the $L_{\text {eff }}\left(L_{R}, c\right)$. This implies that if the TCP goodput performance gain due to $P_{\text {loss }}$ improvement is inadequate to offset the goodput loss due to RTT increase, then the LL ARQ scheme will achieve less TCP goodput compared to the FP SMD scheme. In other words, if $\left(P_{\text {Loss }}^{(l l)}, R T T^{(l l)}\right)$ and $\left(P_{\text {loss }}^{(f p)}, R T T^{(f p)}\right)$ are the $P_{\text {loss }}$ and $R T T$ for the LL ARQ SMD and FP SMD schemes, respectively, at a given SNR, then, from (8), it can be seen that LL ARQ SMD will provide more TCP throughput/goodput than that of the FP SMD if

$$
\left(\frac{P_{l o s s}^{(f p)}}{P_{l o s s}^{(l l)}}\right)>\left(\frac{R T T^{(l l)}}{R T T^{(f p)}}\right)^{2}
$$

and less otherwise. This performance behaviour is illustrated in Fig. 6 where the TCP goodput is plotted for both the schemes. From Fig. 6, we observe the following: 1) LL ARQ SMD gives significantly higher TCP goodput than FP SMD for SNRs above $6.5 \mathrm{~dB}$. This is because at these SNRs the link quality is reasonably good so that $L_{e f f}$ for LL ARQ SMD is less than $L$, and this larger $L$ keeps the maximum achievable throughput to a smaller value (i.e., $\mu / L$ ) for FP SMD. 2) In the SNR range from 2 to $6 \mathrm{~dB}$, however, the FP SMD offers higher goodput than LL ARQ SMD. This is because in this low SNR region, the average number of retransmission attempts in LL ARQ SMD (i.e., $L_{\text {eff }}$ ) becomes larger than $L$ which makes FP SMD offer higher goodputs.

The above observations suggest that the design of a hybrid scheme which adaptively switches between LL ARQ SMD and FP SMD depending on the channel conditions is desired. We present such a hybrid scheme in the following subsection. 


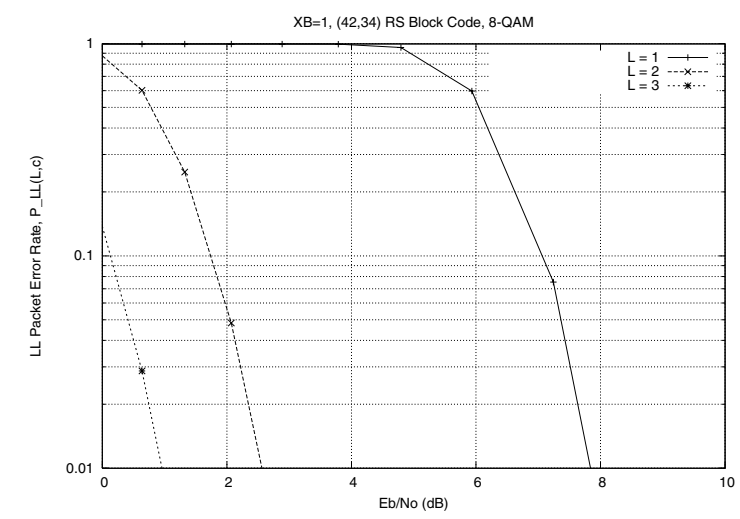

Fig. 7. LL packet error rate, $P_{L L}(L, c)$ versus $E_{b} / N_{0} . X_{B}=1$. $(42,34)$ RS block code. 8-QAM.

\section{Hybrid SMD Scheme}

Here, we present a hybrid scheme which adaptively switches between LL ARQ SMD and FP SMD schemes depending on measured LL packet error rate. Fig. 7 shows the LL packet error rate, $P_{L L}(L, c)$, as a function of SNR for $L=1,2,3$ for the same system parameters in Fig. 6. An estimate of this LL packet error rate can be obtained at the receiver by counting the number of LL packets in error over a certain number of TCP packets. It is desired that this estimate is accurate even when the measurement is performed over small number of TCP packets. In order to achieve this, we propose to count the errors in the first attempt LL packets (i.e., $L=1$ or $L_{R}=1$ ). It has the following advantages; first, the second or third attempts may not happen for a given LL packet (e.g., in case of a LL packet success in the first attempt itself), whereas the first attempt of a LL packet always happens, and second, the error probabilities for the second or third attempts are too small to make reasonably accurate estimate in a reasonable time. On the other hand, as can be seen from Fig. 7, $P_{L L}(L, c)$ for $L=1$ (i.e., error rate of first attempt LL packets) are high enough to make an accurate estimate of $P_{L L}(1, c)$ within a few TCP packets, particularly in the SNR regions where mode change can be beneficial. For example, $P_{L L}(L, c)$ is about 0.6 for $\mathrm{SNR}=6 \mathrm{~dB}$, implying that, for the system with parameters as in Fig. 6, the mode can be set to FP SMD if more than 30 out of $X_{L}=48$ first attempt LL packets in a TCP packet are found to be in error, and stay in LL ARQ SMD mode otherwise.

Two-Mode Hybrid SMD System: We have analyzed such a hybrid adaptive scheme with two modes of operation. In Mode 1 , the system operates in LL ARQ SMD with $L_{R}=4$, and in Mode 2 it operates in FP SMD with $L=2$. The system operates in Mode 2 as long as the number of first attempt LL packet errors fall in the range $n_{1}$ to $n_{2}$ in $n_{T}$ number of TCP packets; the system operates in Mode 1 otherwise. We assume that even in the FP SMD (Mode 2) transmission, LL packet errors will be counted (for which CRC bits are assumed in the 1 st transmission of a TCP packet). The range $\left(n_{1}, n_{2}\right)$ can be chosen based on the SNR range and the corresponding $P_{L L}(1, c)$ range over which the mode change is desired. For this hybrid system, we have derived the expressions for average $P_{\text {loss }}$ and $R T T$ and used these expressions in the square-root formula and computed the TCP goodput.
1) Design Example and Performance:: In Fig. 8, we present a comparison of the TCP goodput performance of the three different schemes, namely, a) LL ARQ SMD with $L_{R}=4$, b) FP SMD with $L=2$, and $c$ ) Hybrid SMD scheme with $n_{1}=52, n_{2}=95, n_{T}=2\left(\frac{n_{1}}{n_{T} X_{L}} \approx 0.6\right.$ and $\frac{n_{2}}{n_{T} X_{L}} \approx 0.99$ which is the range of $P_{L L}(1, c)$ values around which switch to Mode 2 is beneficial). It can be seen that the Hybrid SMD scheme retains the performance of LL ARQ SMD in the SNR ranges $>6 \mathrm{~dB}$ and $<3.75 \mathrm{~dB}$. In the $\mathrm{SNR}$ range $3.75 \mathrm{~dB}$ to 6 $\mathrm{dB}$, the hybrid scheme results in better performance than the LL ARQ SMD scheme (it achieves performance close to the FP SMD scheme), illustrating the benefit of mode adaptation.

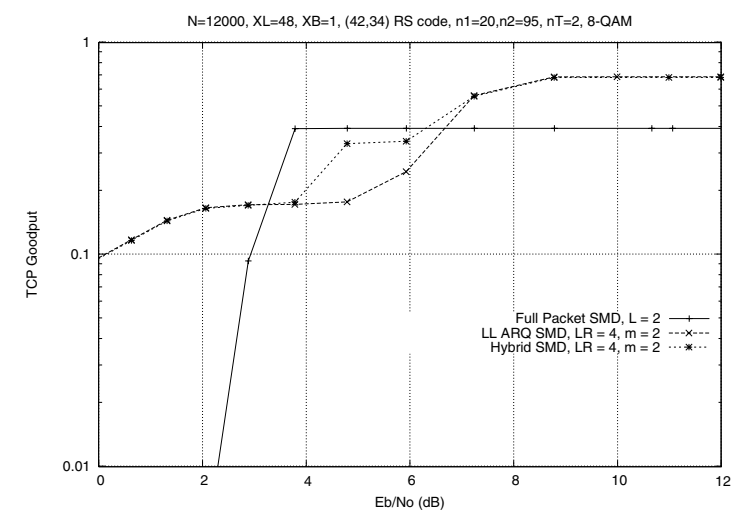

Fig. 8. TCP goodput versus $E_{b} / N_{o}$ for $a$ ) Full Packet SMD, $b$ ) LL ARQ SMD, and $c$ ) Hybrid SMD. $N=12000, H_{T}=160, N_{L}=252, H_{L}=$ $24, X_{L}=48, X_{B}=1, \alpha=1.5, q=10$. 8-QAM, (42,34) RS block code, $n_{1}=52, n_{2}=95, n_{T}=2$. Prop. \& proc. delay slots on wireless, $m=2$.

\section{Simulations}

In the TCP goodput analyses in the previous section, we have used the square-root formula (Eq. (8)) for the TCP throughput, which is only approximate. Particularly, it ignores TCP timeouts. Also, only an upper bound on the QAM symbol error rate (Eq.(3)) is used in the analysis. It would be of interest to see the effect of these two approximations on the analytical performance prediction. Accordingly, we carried out the performance evaluation using simulations as well. We used $n s$ (network simulator) for TCP level simulation and Matlab for SMD/LL ARQ level simulation [4]. We obtained packet error traces and effective LL packet length traces from the Matlab simulation and used these trace files as input files to the $n s$ simulation. We observed that the analytical results closely match the simulation results at high SNRs. At low SNRs, the analysis slightly overestimates the goodput performance, which is expected since timeouts can occur frequently at low SNRs which is ignored by the square-root formula. We note that this work can be extended to fading/diversity channels.

\section{REFERENCES}

[1] H. Samra, Z. Ding, and P. M. Hahn, "Optimal symbol mapping diversity for multiple packet transmissions," Proc. IEEE ICASSP'2003, pp. 181-184, 2003.

[2] J. V. K. Murthy and A. Chockalingam, "Log-likelihood ratio based optimum mappings selection for symbol mapping diversity with $M$ QAM,'Proc. NCC'2005, Kharagpur, India, January 2005.

[3] T. V. Lakshman and U. Madhow, "The performance of TCP/IP for networks with high bandwidth-delay products and random loss," IEEE/ACM Trans. on Networking, June 1997.

[4] P. Ramdoot Kumar, "Cross-layer designs in wireless networks using symbol mapping diversity," M.E Project Mid-term Report, Dept. of ECE, Indian Institute of Science, Bangalore, January 2005. http://wrl.ece.iisc.ernet.in/Prep.html 